\title{
Electron-ion coupling in semiconductors beyond Fermi's golden rule
}

\author{
Nikita Medvedev, ${ }^{1,2,3,{ }^{*}}$ Zheng Li, ${ }^{4,1}$ Victor Tkachenko, ${ }^{1}$ and Beata Ziaja ${ }^{1,5}$ \\ ${ }^{1}$ Center for Free-Electron Laser Science, Deutsches Elektronen-Synchrotron DESY, Notkestrasse 85, D-22607 Hamburg, Germany \\ ${ }^{2}$ Department of Radiation and Chemical Physics, Institute of Physics, Czech Academy of Sciences, \\ Na Slovance 2, 18221 Prague 8, Czech Republic \\ ${ }^{3}$ Laser Plasma Department, Institute of Plasma Physics, Czech Academy of Sciences, Za Slovankou 3, 182 00 Prague 8, Czech Republic \\ ${ }^{4}$ SLAC National Accelerator Laboratory, 2575 Sand Hill Road, Menlo Park, California 94025, USA \\ ${ }^{5}$ Institute of Nuclear Physics, Polish Academy of Sciences, Radzikowskiego 152, 31-342 Kraków, Poland \\ (Received 11 October 2016; revised manuscript received 21 December 2016; published 31 January 2017)
}

\begin{abstract}
In the present work, a theoretical study of electron-phonon (electron-ion) coupling rates in semiconductors driven out of equilibrium is performed. Transient change of optical coefficients reflects the band gap shrinkage in covalently bonded materials, and thus, the heating of atomic lattice. Utilizing this dependence, we test various models of electron-ion coupling. The simulation technique is based on tight-binding molecular dynamics. Our simulations with the dedicated hybrid approach (XTANT) indicate that the widely used Fermi's golden rule can break down describing material excitation on femtosecond time scales. In contrast, dynamical coupling proposed in this work yields a reasonably good agreement of simulation results with available experimental data.
\end{abstract}

DOI: 10.1103/PhysRevB.95.014309

\section{INTRODUCTION}

Since the last decade, various reports on experimental studies of electron-ion coupling in highly excited materials point to their striking disagreement with theoretical predictions [1,2]. Different theoretical models overestimate the experimentally observed heating rates by a factor of up to a few orders of magnitude. The rates do then often need to be adjusted to the experimental data [2,3]. Such a disagreement motivated $\mathrm{Ng}$ to list the problem of inhibited electron-ion coupling among the "outstanding questions" in the field of warm dense matter [1]. This problem, however, is not limited to the warmdense-matter regime only, but affects also laser-excited solids which indicates its general significance. Different extensions of the standard electron-phonon coupling approach have been proposed recently to improve its agreement with experimental data in metals, see, e.g., Refs. [4-7]. In semiconductors or dielectrics, the details of electron-phonon coupling are even less known, in spite of devoted theoretical efforts such as reported, e.g., in Refs. [8-10].

Experimentally, it is not straightforward to monitor the electron-ion coupling rate with a sufficient temporal resolution since individual electron-ion scattering acts occur at femtosecond time scales $[11,12]$. Most of the experimental studies only infer average heating rates without following their temporal evolution during the relaxation stages of a strongly excited system [11-15].

A system under irradiation with an ultrashort laser pulse undergoes a sequence of excitation and relaxation processes. Electrons after photoabsorption are transiently excited, forming a nonequilibrium distribution which is typically relaxing at femtosecond time scales [16]. The thermalized electrons retain high temperature. They then exchange their energies with ions. Significant heating of ions via electron-phonon coupling requires typically a time scale of a few picoseconds [17]. At the same time, in covalently bonded semiconductors electronic

*nikita.medvedev@fzu.cz excitation can lead to nonthermal phase transitions (such as melting) induced by the modification of the interatomic potential for sufficiently high fluences [18-20]. After the local equilibration of electron and ion temperature, the ionic system is still hot. Further relaxation may lead to observable material modifications: structural phase transitions, formation of warm dense matter or even plasma, ablation, etc. [21-24]. The stage when the exchange of energy between the thermalized electrons and lattice occurs is of the main interest for the present study.

In this work we test the recently developed tight-bindingbased approach for nonadiabatic coupling between electrons and atoms [25]. In particular, we analyze the applicability of Fermi's golden rule (FGR) to materials excited at femtosecond time scales, and compare it to a more general dynamical coupling (DC) scheme within the first order approximation [26]. It was indicated, for example, in Ref. [27] that FGR may overestimate electron-ion coupling rates in plasma. Here we demonstrate that FGR, in general, is inapplicable for the description of electron-ion coupling at femtosecond time scales, i.e., shorter than the characteristic time scales of the electron-phonon interaction in solids.

FGR approach is widely used in the studies of femtosecond electron kinetics in irradiated solids. It was employed in a variety of approaches such as two-temperature model and its extensions [2,3], Boltzmann equation [16,28-31], semiconductor Bloch equations (SBEs) [32-34], and others. Therefore, it is of a crucial importance to be aware of its applicability restrictions. At ultrashort time scales one can use instead the dynamical description appropriate in this regime.

It has been known for some time that transient changes of optical properties may carry information on the undergoing changes of electronic and atomic structure of irradiated materials $[21,35,36]$. Recently we pointed out how exactly the pump-probe experiments measuring optical properties of semiconductors can access the information on the electron-ion coupling rates in semiconductors [37]. This is possible due to the effect of the band gap shrinkage (progressing with the increase of the ion temperature) on the optical properties of 
the semiconductor. It results in their so-called "overshooting." For example, the optical reflectivity may not return to its original value (before the excitation) but raises above it on a picosecond time scale [35,36]. Consequently, with the reflectivity measurements the heating of ions coupled to a hot electronic ensemble can be accessed [37]. Currently, dedicated pump-probe experiments can follow transient changes of the optical properties of semiconductors with a few-femtosecond resolution $[21,38,39]$.

\section{MODEL}

In order to study electron-ion coupling in semiconductors, our hybrid simulation tool XTANT has been used [20,25]. This recently developed model consists of: (i) a Monte Carlo (MC) code tracing highly excited nonequilibrium electrons emitted after exposure of a material to a free-electron laser pulse; (ii) thermodynamic approach to evaluate low-energy electron distribution within the valence and at the bottom of the conduction band; (iii) Boltzmann collision integral for calculations of electron-ion energy exchange; and (iv) tight-binding (TB) molecular dynamics (MD) modeling the atomic motion on a changing potential energy surface. The potential energy surface is affected by the transient state of the electron distribution function, and by the positions of all atoms in the simulation box, with periodic boundary conditions imposed [20]. It also depends on the transient electron eigenstates, and thus changes during the nonthermal phase transition; these effects are implicitly included into the tight-binding MD model, as it was discussed in detail, e.g., in Ref. [24].

In case of optical-pulse irradiation, photoabsorption proceeds via excitation of valence or conduction-band electrons. Radiation can also be absorbed directly by free carriers. Thus, during the laser pulse, the electron distribution function looks differently from the discussed case of x-ray irradiation. However, as it quickly relaxes to the equilibrium Fermi-Dirac distribution, there is no fundamental difference between the later relaxation of an optically or x-ray excited electronic system after the triggering laser pulse and secondary electron cascades are over, provided that the absorbed dose is the same in both cases [40]. That is also the electron-phonon coupling acts identically in both cases.

Optical properties are calculated from the complex dielectric function [CDF, $\epsilon(\omega)]$ within the random-phase approximation following Ref. [41]:

$$
\epsilon^{\alpha \beta}(\omega)=\delta_{\alpha, \beta}+\frac{e^{2} \hbar^{2}}{m^{2} \Omega e_{0}} \sum_{i j} \frac{F_{i j}}{E_{i j}^{2}} \frac{f_{e}\left(E_{j}\right)-f_{e}\left(E_{i}\right)}{\hbar \omega-\hbar \omega_{i j}+i \hbar \gamma},
$$

where $\Omega$ is the volume of the supercell, $m$ is the mass of a free electron, $\epsilon_{0}$ is the vacuum permitivity, and $\hbar$ is Planck's constant. $\hbar \omega_{i j}=E_{j}-E_{i}$ is transition energy between two eigenstates $|i\rangle$ and $|j\rangle, f_{e}\left(E_{i}\right)$ and $f_{e}\left(E_{j}\right)$ are the corresponding transient occupation numbers (electron distribution function), $\omega$ is the frequency as a variable in the complex dielectric function, and $F_{i j}=|\langle i|\hat{p}| j\rangle|^{2}$ is the oscillator strength, obtained within Trani's formalism for tight-binding calculations [41]. As it was demonstrated in Ref. [40], this method can satisfactorily trace transient optical properties of excited solids during phase transitions.

In Eq. (1), $\gamma$ is the inverse electron relaxation time chosen to be $\gamma=1.5 \times 10^{13} \mathrm{~s}^{-1}$ [40]. Strictly speaking, in the RPA approximation this parameter should tend to zero, and is kept finite here for numerical purposes only. Its finite value does not affect the results except for broadening of the peaks in the CDF [40]. It is well known that within the RPA approximation, the electronic collisions cannot be treated consistently [42]. In order to introduce electronic collisions into the model, one has to go beyond the RPA framework (e.g., apply the LindhardMermin dielectric function $[43,44]$ ), which is beyond the scope of the present work.

Nonadiabatic electron-ion coupling is introduced in XTANT via Boltzmann collision integral $I_{i, j}^{e \text {-at }}$ :

$$
I_{i, j}^{e-\text { at }}=w_{i, j}\left\{\begin{array}{l}
f_{e}\left(E_{i}\right)\left[2-f_{e}\left(E_{j}\right)\right]-f_{e}\left(E_{j}\right)\left[2-f_{e}\left(E_{i}\right)\right] G_{\mathrm{at}}\left(E_{i}-E_{j}\right), \text { for } i>j, \\
f_{e}\left(E_{i}\right)\left[2-f_{e}\left(E_{j}\right)\right] G_{\mathrm{at}}\left(E_{j}-E_{i}\right)-f_{e}\left(E_{j}\right)\left[2-f_{e}\left(E_{i}\right)\right], \text { for } i<j,
\end{array}\right.
$$

where $w_{i, j}$ is the rate for an electron transition between the energy levels $i$ and $j$; here $f_{e}\left(E_{i}\right)$, a transient electron distribution function, is assumed to be a Fermi-Dirac distribution. It defines electron population on the energy level $E_{i}$ (eigenstate of the transient TB Hamiltonian), and $G_{\text {at }}(E)$ is the integrated Maxwellian function for atoms [25].

Knowledge of the collision integral allows one to evaluate the energy flux between electrons and ions at each time step:

$$
Q=\sum_{i, j} I_{i, j}^{e-\text { at }} E_{i}
$$

where the summation is running through all the electronic orbitals for transitions between each pair of levels [25]. The transferred energy is then distributed among all the atoms in the simulation box by the appropriate velocity scaling.

\section{A. Electron-ion energy exchange rate}

Let us briefly recall the quantum mechanical theory of the probability of a transition between two states. Generally, the probability of an electron transition between two states (in our case mediated by an ion displacement) during a time $\delta t$ can be written within the first order approximation as follows [26]:

$$
P_{i, j}=|\langle i(t) \mid j(t+\delta t)\rangle|^{2},
$$

where $\langle i(t)|$ and $|j(t+\delta t)\rangle$ are the $i$ th and $j$ th eigenfunctions of the Hamiltonian at the time instants $t$ and $t+\delta t$, respectively. It reflects the fact that the probability of the transition is determined by the change of the system state during the given time interval. To obtain the transition rate, a derivative of this matrix element should be taken, which results in the following 
expression suitable for finite-difference implementation:

$$
w_{i, j}=|[\langle i(t) \mid j(t+\delta t)\rangle-\langle i(t+\delta t) \mid j(t)\rangle] / 2|^{2} \frac{1}{\delta t} .
$$

Details of the derivation of this expression are given in Appendix A.

If the Hamiltonian is time independent, the eigenfunctions can be expressed as plane waves without explicit time dependence. Under the assumption of periodic atomic motion within a harmonic potential with the frequency of $\omega_{\mathrm{ph}}$ (phonon frequency), the time derivative of Eq. (4) yields the following scattering rate:

$$
w_{i, j}=\frac{2}{\hbar^{2}}\left|M_{e-\mathrm{at}}\left(E_{i}, E_{j}\right)\right|^{2} \frac{\sin \left[\left(\omega_{i j}-\omega_{\mathrm{ph}}\right) \delta t\right]}{\omega_{i j}-\omega_{\mathrm{ph}}},
$$

where again $\omega_{i j}=\left(E_{i}-E_{j}\right) / \hbar$ and the matrix element for electron-ion scattering $M_{e \text {-at }}\left(E_{i}, E_{j}\right)=$ $[\langle i(t) \mid j(t+\delta t)\rangle-\langle j(t) \mid i(t+\delta t)\rangle]\left(E_{j}-E_{i}\right) / 2$. It was derived in Ref. [25] and includes the overlap of electronic wave functions. In case of harmonic ion displacement of small amplitude around the equilibrium positions, $M_{e \text {-at }}$ reduces to the conventional form of electron-phonon coupling matrix element in the Debye-Hückel form [45].

Note that Eq. (6) has an explicit time dependence within the sine function. This time dependence can be erased, assuming an instant scattering event (Markov process). Physically, this assumption holds at time scales much longer than the duration of an individual scattering event, by setting $\delta t \rightarrow \infty$. In this case, the probability reduces to the well-known Fermi's golden rule [26]:

$$
w_{i j}=\frac{2 \pi}{\hbar^{2}}\left|M_{e-\mathrm{at}}\left(E_{i}, E_{j}\right)\right|^{2} \delta\left(\omega_{i j}-\omega_{\mathrm{ph}}\right),
$$

with $\delta(x)$ being the Dirac's delta function.

However, for the femtosecond time scales we consider here, e.g., in the case of femtosecond-laser-pulse irradiation, the assumptions leading to the FGR are not satisfied because [24]:

(i) In case of strong electronic excitation, atoms might experience rapid modifications of the potential energy surface, leading to an anharmonic atomic motion and significant atom displacements. In an extreme case, they can induce nonthermal melting or solid-to-solid phase transition, breaking the crystal symmetry [24]. That implies that the periodic harmonic motion [phonons, Eq. (6)] cannot be assumed.

(ii) Each individual electron-phonon scattering event in a solid lasts for a time span that can be estimated by the inverse phonon frequency (phonon absorption or emission which takes, typically, a few tens to a hundred of femtoseconds $t_{\mathrm{ph}} \sim$ $\left.1 / \omega_{\text {ph }}\right)$ [46-48]. Thus, an instant electron-phonon collision [FGR, Eq. (7)] cannot be assumed, as was also reported earlier, see, e.g., [49].

Therefore, strictly speaking, neither phononic approximation nor Fermi's golden rule is applicable for modeling of the material excitation induced by intense femtosecond laser pulses, and the general Eqs. (4) or (5) must be used instead. Consequently, the electron transition rates should be calculated at each time step, accounting for the ongoing changes in the atomic subsystem. The corresponding time interval in Eq. (5), $\delta t$, should be set equal to the time step used in the simulation.
It is typically on an attosecond time scale, as will be discussed in the next section.

The dynamical coupling rate, Eq. (5), includes explicit dependence on time without additional assumptions of long time scales and harmonic atomic oscillations. It thus accounts for the evolution of the system during an ongoing individual "collision" and the induced energy exchange.

Although FGR is convenient for estimation of transition probabilities (since it does not have an explicit time dependence, and thus does not require time-dependent calculations), it can overestimate the transition rates on short time scales. Application of FGR to describe electron-phonon scattering in an electronic system out of equilibrium undergoing rapid changes on a femtosecond time scale, i.e., beyond the limit of validity of FGR, could be one of the reasons for the disagreement of theoretical results with the "inhibited" electron-ion coupling observed experimentally $[1,12,27]$.

Note, however, that Eq. (5) does not have the energy conservation built-in. Electrons do not have to populate only the electronic orbitals [energy levels in Eqs. (2) and (5)], but can also transiently depart from them. In order to incorporate the full dynamics, the time-dependent Schrödinger equation should be used. However, it is computationally too costly for implementation. Thus, the electrons are assumed to always populate the electronic orbitals, and only transitions between them are allowed in the simulation, similar to $a b$ initio femtochemical models [50,51].

For wide-band-gap materials (e.g., diamond in the present study), electron transitions across the band gap are excluded from the collision integral Eq. (2). This is a standard technique used in the $a b$ initio femtochemistry [52,53]. This restriction is necessary, as an atomic system cannot accept arbitrarily large amount of energy due to electronic transition. This would violate the energy and momentum conservation. In the present calculations, the transitions between the energy levels separated by more than $5 \mathrm{eV}$ are excluded. The simulation results are not sensitive to the particular choice of this acceptance window within the range from $\sim 3$ up to $\sim 6 \mathrm{eV}$ in case of diamond. For the acceptance windows larger than $\sim 6 \mathrm{eV}$, some transitions across the band gap start to contribute which makes the coupling in diamond too fast. For the acceptance windows smaller than $\sim 3 \mathrm{eV}$, some transitions within the bands are missing, artificially slowing down the energy exchange. Thus, the $5 \mathrm{eV}$ window is used throughout the work for diamond. In the case of silicon and graphite, which have small band gaps, the choice of the cutoff for the acceptance window does not affect the predictions, obtained for the excitation and the corresponding electron temperature regime studied here. The same acceptance windows of $5 \mathrm{eV}$ is used for all materials.

\section{RESULTS}

\section{A. Convergence study}

In the calculations performed with XTANT model, we noticed that using the FGR, Eq. (7), produces strongly nonconvergent results: the heating rate calculated depends on the time step chosen. This directly contradicts the assumptions necessary for the derivation of the FGR. 


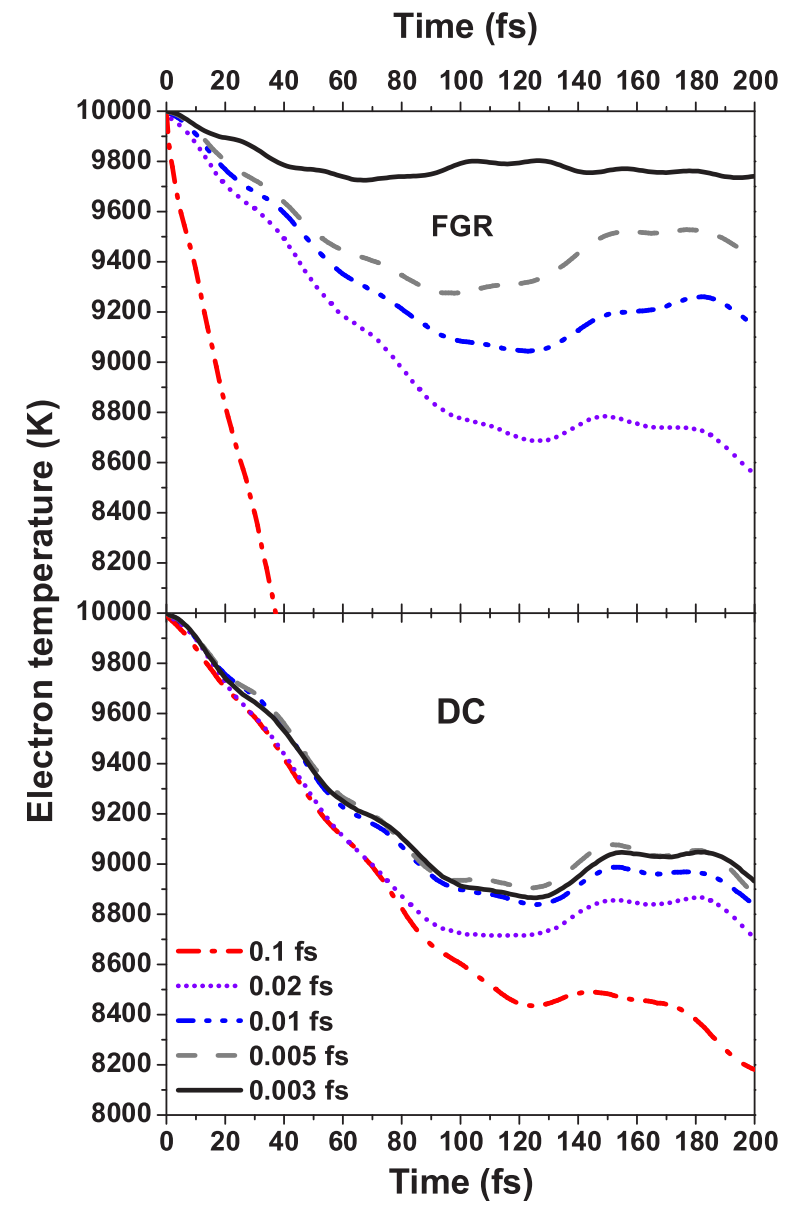

FIG. 1. Convergence study for transient changes of electronic temperature due to the electron-ion energy exchange in silicon. The changes were calculated with Fermi's golden rule [FGR, Eq. (7), top panel] or a dynamical coupling approach [DC, Eq. (5), bottom panel]. Results obtained with different time steps used are compared.

Starting from the time steps of 0.1 fs down to 3 as, no convergence was found in the case of FGR calculations, as illustrated in Fig. 1 for silicon. Here in all cases the initial electron temperature was set to $T_{e}=10000 \mathrm{~K}$ and the ion temperature $T_{i}=300 \mathrm{~K}$.

The FGR calculations do not converge due to the fact that the assumptions of time independence of the Hamiltonian and the corresponding wave functions [points (i) and (ii) in the previous section], necessary for the validity of FGR, break down. The system's state is changing at each time step, which makes the matrix element $M_{e \text {-at }}$ time dependent.

In contrast, the calculations using the dynamical coupling (DC) expression for electron-ion coupling, Eq. (5), converged for the time steps of $\sim 0.01 \mathrm{fs}$, see also Fig. 1. Discrepancies for the calculated electron temperatures with the time steps of below $\sim 0.02$ fs are marginal.

The results suggest that our earlier work [25] which used FGR with the time step of $0.1 \mathrm{fs}$ might have underestimated the time scales of the thermal melting of silicon. It seems to take longer than previously reported; however, the values of the damage threshold and the conclusions drawn remain unaffected by the modified time scales (see Appendix B). That is because the damage depends on the total deposited

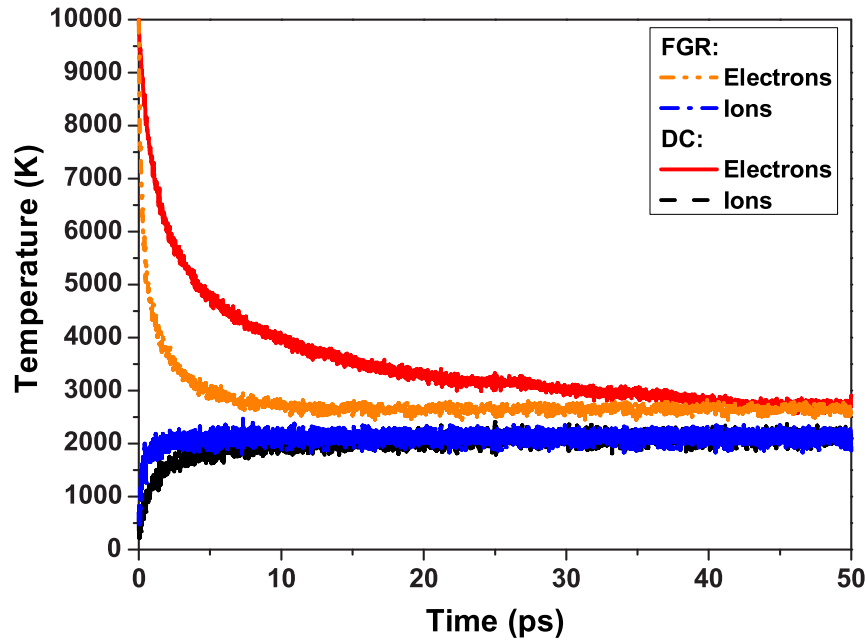

FIG. 2. Evolution of electron and ion temperatures in silicon calculated with electron-ion coupling obtained with Fermi's golden rule [FGR, Eq. (7), using time step of $0.1 \mathrm{fs}$ ], and the dynamical coupling (DC) approach, Eq. (5).

energy, which is the same in both cases. Without additional channels for energy loss, such as heat diffusion, which were not included in the model, a change of the time scale for energy delivery into the atomic system does not affect the predicted damage threshold.

To compare the relaxation time scales, Fig. 2 shows the electron-ion thermalization for silicon calculated within the FGR [Eq. (7) using the time step of $0.1 \mathrm{fs}$ ] and the DC [Eq. (5) with the time step of $0.01 \mathrm{fs}$ for convergence]. The FGR calculations with the time step of $0.1 \mathrm{fs}$ as used in the previous work, Ref. [25], are shown here only for comparison. We can see that the FGR (with the time step of $0.1 \mathrm{fs}$ ) significantly overestimates the time scale for electron-ion energy relaxation.

In both simulations, the equilibration of temperatures is not exact, as it was also noted in Ref. [25]. This is due to the finite band gap in silicon, and to the available numerical accuracy. The same effect was observed for diamond and graphite (not shown).

All further calculations reported below are performed using the dynamical coupling approach with the time step of $0.01 \mathrm{fs}$.

\section{B. Effect of electron-ion coupling on optical properties}

In order to compare the modeled dynamical coupling to experimental data, the effect of the electron-ion coupling on the optical properties is analyzed. The calculations are performed for the following pulse and dose parameters in irradiated silicon, corresponding to the pump-probe experiments from Ref. [21]: absorbed dose of 1.3 times the damage threshold (producing $\sim 0.78 \mathrm{eV} /$ atom [25]), Gaussian pulse duration of $60 \mathrm{fs}$ FWHM, and probe pulse of $625 \mathrm{~nm}$ under $70.5 \%$ incidence.

Although the pump-photon energy modeled here is $30 \mathrm{eV}$, while in Ref. [21] an optical pump was used in the experiment, we can compare the results of our simulations with the experimental data, expecting only slight deviations during the pulse, as discussed above [40]. Already by the end of the pulse, one can expect that the simulation and the experimental 


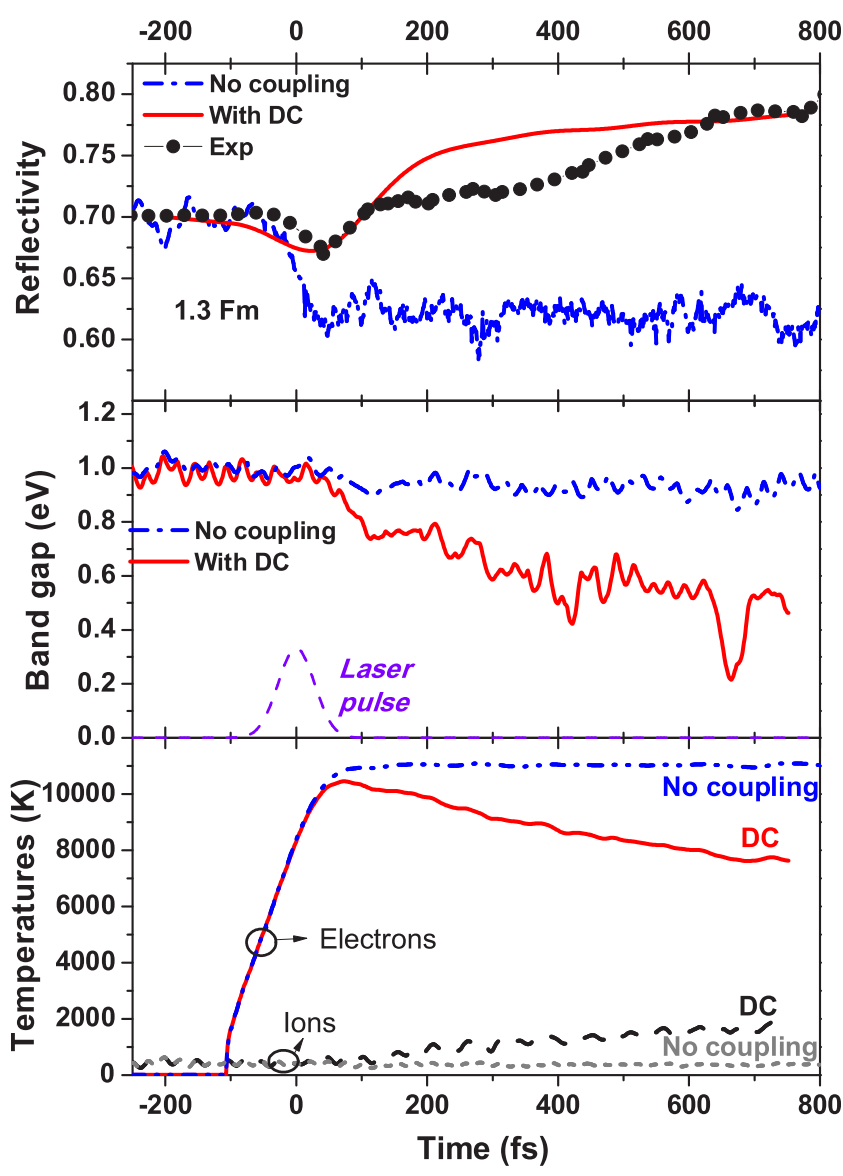

FIG. 3. Top panel: Optical reflectivity of silicon at $625 \mathrm{~nm}$ probe wavelength under $70.5^{\circ}$ incidence. Calculations with XTANT are shown, using (i) the dynamical coupling approach, Eq. (6), red line; and (ii) no coupling included (Born-Oppenheimer approximation), blue dash-dotted line. Experimental data from Ref. [21] are shown for comparison (black circles). Middle panel: Evolution of the band gap of silicon simulated with and without nonadiabatic electron-ion coupling. Laser pulse shape is schematically shown with a violet dashed line. Bottom panel: Respective electron and ion temperatures.

conditions should approach each other, as demonstrated in Ref. [40], thus a meaningful comparison can be made. This is due to the similar final electronic states of the system achieved after the excitation with both pulses in which fluences were adjusted to ensure the same absorbed dose per atom; after the electrons thermalize, the two cases become identical.

For $30 \mathrm{eV}$ photons, only electrons of $<30 \mathrm{eV}$ energies are excited. Their relaxation towards the bottom of the conduction band takes only a few collisions and accomplishes within about a femtosecond [54]. Note that in Ref. [25], photon energy of $1000 \mathrm{eV}$ was used, for which the electron cascades are significantly longer (few tens to a hundred fs [54]).

Transient values of the optical reflectivity are shown in Fig. 3; for better comparison with experimental data, they were smoothed by a convolution with the probe pulse of a finite duration (60 fs FWHM) [21,40]. We can see in Fig. 3 that the reflectivity calculated with dynamical coupling is reasonably close to the experimental data.

One can see that the optical reflectivity directly depends on the band gap. When the band gap is shrinking due to the increase of ion temperature (see middle and bottom panels of Fig. 3, respectively), the reflectivity is changing accordingly.

Figure 3 also demonstrates that using Born-Oppenheimer approximation that excludes electron-ion coupling (marked as "no coupling" in Fig. 3) does not lead to the increase of the ionic temperature, as expected [25], and, correspondingly, does not induce band gap shrinkage (in the case of doses below the nonthermal melting threshold). This, in turn, does not trigger any reflectivity increase after the initial drop. The initial drop is caused solely by the electronic excitation. We do not show here the results with FGR as they did not converge.

These results confirm the idea presented in Ref. [37]: the "overshooting effect" observed in experimental data $[35,36]$ is a consequence of the ion heating and the resulting band-gap shrinkage. The optical coefficients such as reflectivity or transmission then encode information on the electron-ion coupling in semiconductors. Thus, the pump-probe experimental schemes can be used to extract the information on electron-ion coupling with femtosecond resolution. Especially the case of FEL-pump optical-probe scheme would be of interest, allowing for a precise control of the dose absorbed during the irradiation $[35,38,39]$, and a uniform volumetric heating of a sample.

Let us mention here that in the case of the absorbed dose above the nonthermal melting threshold, the band-gap collapse can also be induced via nonthermal melting [20,25]. Significant heating of the lattice is then not necessary. For such high doses, the overshooting effect allows us to extract time scales of the predominant nonthermal melting (rather than of the subdominant electron-ion coupling). Such a case was studied in detail in Ref. [40], and will also be discussed here.

The calculated optical reflectivity for different pump-pulse fluences in silicon is shown in Fig. 4. As previously, the data are convolved with a Gaussian probe pulse of $60 \mathrm{fs}$ FWHM corresponding to the experimental conditions of Ref. [21]. Therein, the fluences are given in the units of the damage threshold $F_{m}$. We then compare our results by setting the absorbed dose proportionally to the damage threshold dose, which was previously estimated for $\mathrm{Si}$ to be $\sim 0.6$ $\mathrm{eV} /$ atom [25]. This allows us to avoid any detailed simulations of optical photoabsorption, which is not the focus of the present work.

At high doses above $1.8 F_{m}(\sim 1.17 \mathrm{eV} /$ atom $)$ in the present data set, silicon undergoes nonthermal melting, induced by the modification of the potential energy surface [25]. This is an extremely fast process amorphizing the target within the first $\sim 300-500 \mathrm{fs}$. It is also affecting the optical reflectivity, as one can see in Fig. 4. Similar behavior was reported in Ref. [40]. The thermal contribution to the transition here is only minor, thus, the electron-ion coupling does not affect much the reflectivity changes. However, a slower melting can be observed for lower doses, because the contribution of thermal effects then becomes more significant [25].

For the doses corresponding to $1.5 F_{m}$ and $1.3 F_{m}$, the damage proceeds via thermal melting without significant nonthermal effects. It can then last up to a few picoseconds (compare Fig. 2). Our result obtained for the lowest dose, $1.1 F_{m}$, shows poor agreement with the experimental data. We can identify at least two reasons for that:

(i) In the XTANT hybrid model, the valence and conduction band electrons are assumed to be in a mutual thermal 


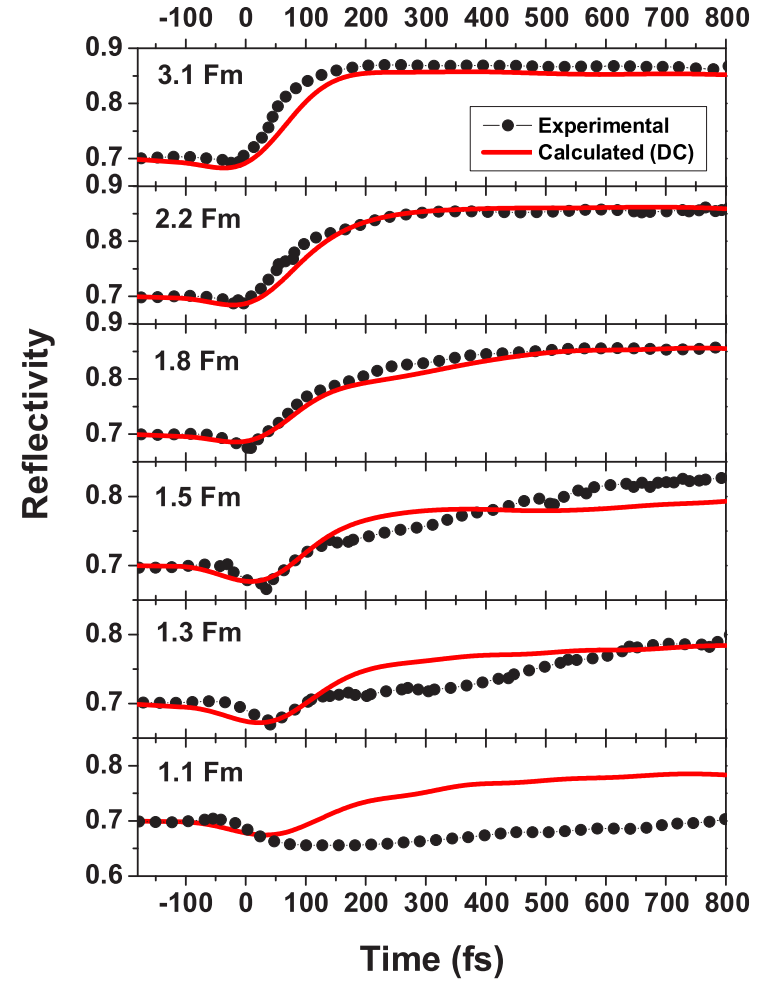

FIG. 4. Comparison of the calculated and experimental [21] reflectivity of silicon at $625 \mathrm{~nm}$ probe wavelength under $70.5^{\circ}$ incidence for different fluences (in the units of damage threshold $F_{m}$ ). Calculations are performed with the dynamical electron-ion coupling, Eq. (6). The data were convolved with a 60 fs Gaussian probe pulse.

equilibrium (following a unified Fermi-Dirac distribution). In the experiment [21] this might not have been the case. The temperature nonequilibrium between valence and conduction band can persist longer for lower pulse fluences [16]. As reported, for example, in Ref. [16], for electrons out of equilibrium, electron-ion (electron-phonon) coupling can be significantly slower. Thus, it can be expected that the temperature-equilibrium-based calculations overestimate ion heating, correspondingly underestimating the time for the reflectivity increase in case of the lowest dose.

(ii) Periodic boundary conditions are used in the calculation, which confine all the absorbed energy inside a supercell. In experiment, this was not the case: heat diffusion may bring some energy out of the irradiated spot. This effect is expected to be more significant at lower fluences, since in this case the damage process takes longer time.

The two assumptions discussed above have always been used in our model, for all studied cases of electron-ion coupling: DC, FGR, or in the scenario with no nonadiabatic coupling. Detailed investigation of the effects beyond these assumptions warrants separate dedicated studies, which are out of the scope of the present work.

\section{DISCUSSION}

Figure 5 shows the calculated dynamical electron-ion coupling parameter in silicon at different deposited doses, corresponding to Fig. 4. The electron-ion coupling parameter \{defined as $g_{e-\text { at }}\left(T_{e}, T_{a}\right)=Q /\left[\left(T_{e}-T_{a}\right) \Omega\right]$, where $Q$ is the

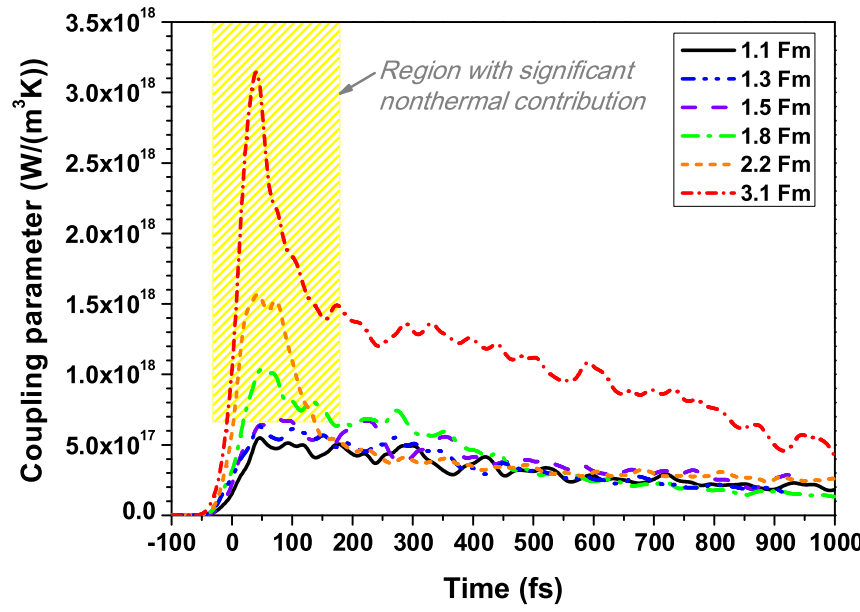

FIG. 5. Evolution of the dynamical electron-ion coupling parameter [Eq. (6)] in silicon irradiated with different pulse fluences.

heat rate defined by Eq. (3), and $\Omega$ is the volume of the simulation supercell $\}$ is a time-dependent function of both electron and ion temperatures.

For the cases below the nonthermal threshold $\left(1.5 F_{m}\right.$ and lower in Fig. 5), an average value of the electronphonon coupling parameter appears to be on the order of $3 \times 10^{17} \mathrm{~W} /\left(\mathrm{K} \mathrm{m}^{3}\right)$ during the first picosecond, as it can be seen in Fig. 5. It is sinking with the decrease of the electron temperature and drops below $10^{17} \mathrm{~W} /\left(\mathrm{K} \mathrm{m}^{3}\right)$ (at $\sim 10 \mathrm{ps,} \mathrm{not}$ shown), which becomes then closer to the values reported in [1], although for a very different parameter range.

The coupling parameter in Fig. 5 nonlinearly increases with the increase of the deposited dose. It always decreases during the cooling of the electron system. For the deposited doses above $1.8 F_{m}$, when nonthermal melting starts to play a role, it exhibits a sudden sharp peak during the first $100 \mathrm{fs}$ after the pulse maximum. The appearance of this peak is due to the linear dependence of $M_{e \text {-at }}$ on the ion velocity, and reflects a strong "softening" of the interatomic potential, in which atoms are gaining the kinetic energy not only via nonadiabatic electron-ion coupling but also as a result of the nonthermal melting-due to modification of the interatomic potential through electronic excitation. This means that the electron-ion coupling parameter in this range should not be interpreted as the thermal electron-phonon coupling, but it contains also a contribution from the nonthermal transition. To emphasize this, the region of significant nonthermal contribution is highlighted in Fig. 5 for the deposited doses above $1.8 F_{m}$. During this time, electrons also reach their highest temperatures. Later, the coupling parameter decreases due to the decrease of the electronic temperature and to the suppression of nonthermal effects. Finally, it approaches an asymptotic value.

Dedicated analysis of a set of simulation runs with different initial electronic and atomic temperatures showed that the electron-ion coupling parameter in silicon scales approximately as $g_{e-\text { at }}\left(T_{e}, T_{a}\right) \sim T_{a} T_{e}^{3}$, within the limits for: (i) the atomic temperature $T_{a}$ from the room temperature up to the melting point $\sim 1687 \mathrm{~K}$, and (ii) for the electronic temperature $T_{e}$ between the room temperature and the limit of a purely nonthermal melting $\sim 17 \mathrm{kK}$. 
Similar calculations were also performed with XTANT for diamond and graphite. The corresponding tight-binding parameters for carbon-based materials are given in Ref. [20]. The simulation results show that in diamond and graphite, an average electron-ion coupling parameters appear to be on the order of $\sim 5 \times 10^{17}$ and $\sim 10^{17} \mathrm{~W} /\left(\mathrm{K} \mathrm{m}^{3}\right)$, respectively. The data reported in Ref. [55], where the coupling parameter in graphite was measured and found to be on the order of $\sim 10^{16} \mathrm{~W} /\left(\mathrm{K} \mathrm{m}^{3}\right)$, could not be directly compared with the present calculations, since the high excitation in those experiments lead to electron energies up to a few $\mathrm{MeV}$. Treatment of such relativistic electrons is beyond reach of our current approach. As electrons of $\mathrm{MeV}$ energies require long times for their thermalization and cascading (roughly on the order of $\sim 35$ ps by nonrelativistic estimation [54]), correspondingly delaying the electron-ion coupling, we expect that this might be one of the reasons why the coupling values reported there are lower than those in the present calculations. Another possibility discussed in the literature is the effect of the coupling of collective atomic and electronic modes that can slow down their energy exchange [27]. Further studies are required to clarify these issues.

\section{CONCLUSIONS}

In this study we confirmed and utilized the earlier observation [37] that a change of optical reflectivity is a consequence of the band gap shrinkage in covalently bonded materials, induced by the heating of the atomic lattice (in case of thermal electron-ion coupling). Using this effect, we studied in detail electron-ion coupling in semiconductors. Dedicated simulations were performed for silicon crystals driven out of equilibrium by a femtosecond laser pulse.

Fermi's golden rule within the phononic approximation (harmonic approximation for the atomic motion) and the proposed alternative approach, dynamical coupling (DC), were analyzed to model the electron-ion coupling in silicon. The observed nonconvergence of the FGR calculations indicates that the widely used phononic approximation and Fermi's golden rule seem to be inapplicable to describe material excitation at femtosecond time scales. This is in contrast to the dynamical coupling which converged for time steps $\sim 0.01 \mathrm{fs}$, and could describe the optical reflectivity measurements with a reasonably good accuracy.

Further studies are required to test the dynamical coupling approach for different materials. However, as the current analysis already indicates that the assumption of an instant scattering event underlying Fermi's golden rule breaks down at femtosecond time scales, and time dependence has then to enter explicitly the electron-ion exchange rates, the proposed
DC approach seems to be a promising alternative to the FGR approximation at those ultrashort time scales.

\section{ACKNOWLEDGMENTS}

The authors thank D. O. Gericke, S. Gorbunov, R. Santra, P. Terekhin, A. E. Volkov, and J. Vorberger for illuminating discussions. Partial financial support from the Czech Ministry of Education (Grants No. LG15013 and No. LM2015083) is acknowledged by N. Medvedev. Z.L. is grateful to the Volkswagen Foundation for financial support via the PaulEwald postdoctoral fellowship.

\section{APPENDIX A: DERIVATION OF THE DYNAMICAL COUPLING TRANSITION RATE}

Electronic transition rate $w_{i, j}(t)$ is defined as a time derivative of the transition probability between the levels $|i\rangle$ and $|j\rangle, P_{i, j}(t)[26,56]:$

$$
w_{i, j}(t)=\frac{d P_{i, j}(t)}{d t} .
$$

Introducing the transition rate

$$
w_{i, j}(t)=\lim _{t \rightarrow t_{0}} \frac{\left[P_{i, j}(t)-P_{i, j}\left(t_{0}\right)\right]}{t-t_{0}}
$$

into the numerical model, we use the finite-difference approximation for the derivative:

$$
w_{i, j}(t) \approx \frac{\left[P_{i, j}(t)-P_{i, j}\left(t_{0}\right)\right]}{\delta t},
$$

where the time step $\delta t \equiv t-t_{0}$ has to be sufficiently short. It corresponds to the time step within our TBMD scheme of XTANT.

As we discretize time into sufficiently short time steps $\delta t$, the interatomic forces are assumed constant during each time step within TBMD part of XTANT. The Hamiltonian does not change during the time step, being updated only at the beginning of the next step. This implies that any time-dependent perturbing potential can be represented as a sequence of steplike functions: constant within any time step and updated before the next one. The corresponding matrix element then reads

$$
V_{i, j}(t)=V_{i, j} \theta\left(t-t_{0}\right),
$$

where $\theta(x)$ is Heaviside step function, and $V_{i, j} \equiv V_{i, j}\left(t_{0}\right)$ is constant during $\delta t$. The perturbation is switched on instantaneously at time $t_{0}$. Consequently, $P_{i, j}\left(t_{0}\right)=0$. The transition rate then reads

$$
w_{i, j}(t)=\frac{P_{i, j}(t)}{\delta t} .
$$

TABLE I. Damage thresholds and transition time scales in diamond calculated with: (i) Born-Oppenheimer (BO) approximation that excludes nonadiabatic coupling; (ii) Fermi's golden rule (FGR) Eq. (7) with the time step of $0.1 \mathrm{fs}$; and (iii) dynamical electron-ion coupling (DC) Eq. (5). Experimental references are also given for comparison wherever available.

\begin{tabular}{lcccc}
\hline \hline & BO & FGR & DC & Experiment \\
\hline Damage threshold (eV/atom) & $0.7-0.75[20,57]$ & $1-1.1$ & $0.65-0.7$ & $\sim 0.7[58]$ \\
Time scales (fs) & $\sim 80-130$ & $\sim 80$ & $\sim 80-150$ & - \\
\hline \hline
\end{tabular}


TABLE II. Damage thresholds and transition time scales in silicon calculated with: (i) Born-Oppenheimer (BO) approximation that excludes nonadiabatic coupling; (ii) Fermi's golden rule (FGR) Eq. (7) with the time step of $0.1 \mathrm{fs}$; and (iii) dynamical electron-ion coupling (DC) Eq. (5). Both damage threshold, for thermal and nonthermal melting, are presented. Experimental references are also given for comparison wherever available.

\begin{tabular}{lcccc}
\hline \hline & BO & FGR & DC & Experiment \\
\hline Thermal melting (TM) (eV/atom) & - & $0.65[25]$ & 0.65 & - \\
Time scales for TM (ps) & - & $\sim 1$ & $\sim 2$ & $1.5-2[59,60]$ \\
Nonthermal melting (NTM) (eV/atom) & $2.1[25]$ & $0.9[25]$ & 0.9 & $\sim 0.9-1[61]$ \\
Time scales for NTM (fs) & $\sim 300[25]$ & $\sim 300-500[25]$ & $\sim 300-1000$ & $300-500[21,61,62]$ \\
\hline
\end{tabular}

We apply the approximate transition probability (for instantaneous approximation consistent with our scheme here) derived in Ref. [26] [Eq. (41.5) therein]:

$$
P_{i, j}(t)=\left|\frac{V_{i, j}}{h \omega_{i, j}}\right|^{2},
$$

where $\omega_{i, j}=\left(E_{i}-E_{j}\right) / \hbar$, and $E_{i}$ are the transient electronic energy levels (band structure). With Eq. (A5) we arrive at the transition rate

$$
w_{i, j}(t)=\frac{1}{\delta t}\left|\frac{V_{i, j}}{h \omega_{i, j}}\right|^{2} .
$$

Utilizing our previously derived finite-difference expression for the perturbation matrix element from Ref. [25]: $V_{i, j}=$ $\left[\left\langle i\left(t_{0}\right) \mid j\left(t_{0}+\delta t\right)\right\rangle-\left\langle i{ }_{0} t+\delta t\right)\left|j\left(t_{0}\right)\right\rangle\right]\left(E_{j}-E_{i}\right) / 2$, we obtain the final expression for the transition rate that has been implemented into XTANT code:

$$
w_{i, j}(t)=\frac{1}{\delta t}\left|\left[\left\langle i\left(t_{0}\right) \mid j\left(t_{0}+\delta t\right)\right\rangle-\left\langle i\left(t_{0}+\delta t\right) \mid j\left(t_{0}\right)\right\rangle\right] / 2\right|^{2} .
$$

\section{APPENDIX B: CALCULATED DAMAGE THRESHOLDS}

Using the dynamical coupling rate from Eq. (5) and the FGR Eq. (7) (with the time step of $0.1 \mathrm{fs}$ ), the following results were obtained for damage thresholds. We sum them up in Tables I and II.

In the case of dynamical coupling, the damage thresholds are lowered for both silicon and diamond, when compared to the results with the Born-Oppenheimer approximation. The effect is more noticeable for silicon, while for diamond it is only minor. Transition time scales with both approaches are similar for diamond, and differing for silicon.

In contrast, FGR coupling in diamond induces much faster heating rates (for the simulation time step of $0.1 \mathrm{fs}$ ), which prevents graphite from forming; this disagrees with the experimental observations of graphitization after an FEL irradiation [58] at the respective threshold fluence.

For silicon the transition time scales obtained with FGR (again with the time step of 0.1 fs to represent earlier works) correspond to those obtained with the Born-Oppenheimer approximation (for the nonthermal melting threshold) and are much shorter than those obtained with the dynamical approach. The experimental damage threshold for nonthermal melting in silicon given in Table II is estimated from the reported threshold density of electron $\sim 6 \%$ in Ref. [61].
[1] A. $\mathrm{Ng}$, Outstanding questions in electron-ion energy relaxation, lattice stability, and dielectric function of warm dense matter, Int. J. Quantum Chem. 112, 150 (2012).

[2] S. Gorbunov, N. Medvedev, P. Terekhin, and A. Volkov, Electron-lattice coupling after high-energy deposition in aluminum, Nucl. Instrum. Methods Phys. Res. Sect. B 354, 220 (2014).

[3] Z. Lin, L. V. Zhigilei, and V. Celli, Electron-phonon coupling and electron heat capacity of metals under conditions of strong electron-phonon nonequilibrium, Phys. Rev. B 77, 075133 (2008).

[4] Y. V. Petrov, N. A. Inogamov, and K. P. Migdal, Thermal conductivity and the electron-ion heat transfer coefficient in condensed media with a strongly excited electron subsystem, JETP Lett. 97, 20 (2013).

[5] L. Waldecker, R. Bertoni, R. Ernstorfer, and J. Vorberger, Electron-Phonon Coupling and Energy Flow in a Simple Metal Beyond the Two-Temperature Approximation, Phys. Rev. X 6, 021003 (2016).
[6] K. P. Migdal, Y. V. Petrov, D. K. Ilnitsky, V. V. Zhakhovsky, N. A. Inogamov, K. V. Khishchenko, D. V. Knyazev, and P. R. Levashov, Heat conductivity of copper in two-temperature state, Appl. Phys. A 122, 408 (2016).

[7] B. I. Cho, T. Ogitsu, K. Engelhorn, A. A. Correa, Y. Ping, J. W. Lee, L. J. Bae, D. Prendergast, R. W. Falcone, P. A. Heimann, J. Daligault, S. Gupta, S. H. Glenzer, J. W. Chan, T. Huser, S. Risbud, R. W. Lee, A. Ng, T. Ao, F. Perrot, M. W. C. Dharmawardana, M. E. Foord, T. Ao, Y. Ping, G. M. Dyer, R. Ernstorfer, A. Mančić, B. I. Cho, T. G. White, Z. Chen, M. W. C. Dharmawardana, F. Perrot, J. Vorberger, D. O. Gericke, T. Bornath, M. Schlanges, U. Reimann, C. Toepffer, A. Ng, P. Celliers, G. $\mathrm{Xu}$, A. Forsman, D. Riley, Z. Lin, L. V. Zhigilei, V. Celli, B. I. Cho, S. Johnson, J. Hohlfeld, S. Wellershoff, J. Güdde, U. Conrad, H. Elsayed-Ali, T. Norris, M. Pessot, W. L. McMillan, G. Grimvall, E. Wohlfarth, L. B. Fletcher, M. G. Gorman, J. Gaudin, F. Dorchies, G. Paolo, D. Prendergast, and G. Galli, Measurement of electron-ion relaxation in warm dense copper, Sci. Rep. 6, 18843 (2016). 
[8] M. V. Fischetti and S. E. Laux, Monte Carlo study of electron transport in silicon inversion layers, Phys. Rev. B 48, 2244 (1993).

[9] N. A. Inogamov, A. Y. Faenov, V. V. Zhakhovsky, T. A. Pikuz, I. Y. Skobelev, Y. V. Petrov, V. A. Khokhlov, V. V. Shepelev, S. I. Anisimov, V. E. Fortov, Y. Fukuda, M. Kando, T. Kawachi, M. Nagasono, H. Ohashi, M. Yabashi, K. Tono, Y. Senda, T. Togashi, and T. Ishikawa, Two-temperature warm dense matter produced by ultrashort extreme vacuum ultraviolet-free electron laser (EUV-FEL) pulse, Contrib. Plasma Phys. 51, 419 (2011).

[10] S. A. Gorbunov, N. A. Medvedev, P. N. Terekhin, and A. E. Volkov, The microscopic model of material excitation in swift heavy ion tracks, Phys. Status Solidi C 10, 697 (2013).

[11] P. Celliers, A. Ng, G. Xu, and A. Forsman, Thermal Equilibration in a Shock Wave, Phys. Rev. Lett. 68, 2305 (1992).

[12] T. G. White, J. Vorberger, C. R. D. Brown, B. J. B. Crowley, P. Davis, S. H. Glenzer, J. W. O. Harris, D. C. Hochhaus, S. Le Pape, T. Ma, C. D. Murphy, P. Neumayer, L. K. Pattison, S. Richardson, D. O. Gericke, and G. Gregori, Observation of inhibited electron-ion coupling in strongly heated graphite, Sci. Rep. 2, 889 (2012).

[13] J. L. Hostetler, A. N. Smith, D. M. Czajkowsky, and P. M. Norris, Measurement of the electron-phonon coupling factor dependence on film thickness and grain size in $\mathrm{Au}, \mathrm{Cr}$, and $\mathrm{Al}$, Appl. Opt. 38, 3614 (1999).

[14] P. E. Hopkins, J. M. Klopf, and P. M. Norris, Influence of interband transitions on electron-phonon coupling measurements in Ni films, Appl. Opt. 46, 2076 (2007).

[15] Z. Li-Dan, S. Fang-Yuan, Z. Jie, and T. Da-Wei, Study on ultra fast nonequilibrium heat transfers in nano metal films by femtosecond laser pump and probe method, Acta Phys. Sin. 61, 134402 (2012).

[16] B. Rethfeld, A. Kaiser, M. Vicanek, and G. Simon, Ultrafast dynamics of nonequilibrium electrons in metals under femtosecond laser irradiation, Phys. Rev. B 65, 214303 (2002).

[17] P. Lorazo, L. J. Lewis, and M. Meunier, Thermodynamic pathways to melting, ablation, and solidification in absorbing solids under pulsed laser irradiation, Phys. Rev. B 73, 134108 (2006).

[18] P. Stampfli and K. H. Bennemann, Dynamical theory of the laserinduced lattice instability of silicon, Phys. Rev. B 46, 10686 (1992).

[19] H. O. Jeschke, M. E. Garcia, and K. H. Bennemann, Microscopic analysis of the laser-induced femtosecond graphitization of diamond, Phys. Rev. B 60, R3701 (1999).

[20] N. Medvedev, H. O. Jeschke, and B. Ziaja, Nonthermal phase transitions in semiconductors induced by a femtosecond extreme ultraviolet laser pulse, New J. Phys. 15, 015016 (2013).

[21] K. Sokolowski-Tinten, J. Bialkowski, and D. von der Linde, Ultrafast laser-induced order-disorder transitions in semiconductors, Phys. Rev. B 51, 14186 (1995).

[22] L. B. Fletcher, H. J. Lee, T. Döppner, E. Galtier, B. Nagler, P. Heimann, C. Fortmann, S. LePape, T. Ma, M. Millot, A. Pak, D. Turnbull, D. A. Chapman, D. O. Gericke, J. Vorberger, T. White, G. Gregori, M. Wei, B. Barbrel, R. W. Falcone, C.-C. Kao, H. Nuhn, J. Welch, U. Zastrau, P. Neumayer, J. B. Hastings, and S. H. Glenzer, Ultrabright $x$-ray laser scattering for dynamic warm dense matter physics, Nat. Photon. 9, 274 (2015).

[23] L. V. Zhigilei, Z. Lin, and D. S. Ivanov, Atomistic modeling of short pulse laser ablation of metals: Connections between melting, spallation, and phase explosion, J. Phys. Chem. C 113, 11892 (2009).

[24] N. Medvedev, V. Tkachenko, and B. Ziaja, Modeling of nonthermal solid-to-solid phase transition in diamond irradiated with femtosecond x-ray FEL pulse, Contrib. Plasma Phys. 55, 12 (2015).

[25] N. Medvedev, Z. Li, and B. Ziaja, Thermal and nonthermal melting of silicon under femtosecond $\mathrm{x}$-ray irradiation, Phys. Rev. B 91, 054113 (2015).

[26] L. D. Landau and L. M. Lifshitz, Quantum Mechanics, Third Edition: Non-Relativistic Theory (Butterworth-Heinemann, London, 1976).

[27] J. Vorberger, D. O. Gericke, T. Bornath, and M. Schlanges, Energy relaxation in dense, strongly coupled two-temperature plasmas, Phys. Rev. E 81, 046404 (2010).

[28] P. B. Allen, Theory of Thermal Relaxation of Electrons in Metals, Phys. Rev. Lett. 59, 1460 (1987).

[29] L. D. Pietanza, G. Colonna, S. Longo, and M. Capitelli, Non-equilibrium electron and phonon dynamics in metals under femtosecond laser pulses, Eur. Phys. J. D 45, 369 (2007).

[30] N. S. Shcheblanov and T. E. Itina, Femtosecond laser interactions with dielectric materials: Insights of a detailed modeling of electronic excitation and relaxation processes, Appl. Phys. A 110, 579 (2012).

[31] B. Y. Mueller and B. Rethfeld, Relaxation dynamics in laserexcited metals under nonequilibrium conditions, Phys. Rev. B 87, 035139 (2013).

[32] S. Haas, F. Rossi, and T. Kuhn, Ultrafast Coherent and Incoherent Dynamics in Photoexcited Semiconductors (Springer, New York, 1995), pp. 473-476.

[33] J. Shah, Ultrafast Spectroscopy of Semiconductors and Semiconductor Nanostructures, Vol. 115 of Springer Series in Solid-State Sciences (Springer, Berlin, 1999).

[34] F. Rossi and T. Kuhn, Theory of ultrafast phenomena in photoexcited semiconductors, Rev. Mod. Phys. 74, 895 (2002).

[35] T. Maltezopoulos, S. Cunovic, M. Wieland, M. Beye, A. Azima, H. Redlin, M. Krikunova, R. Kalms, U. Frühling, F. Budzyn, W. Wurth, A. Föhlisch, and M. Drescher, Single-shot timing measurement of extreme-ultraviolet free-electron laser pulses, New J. Phys. 10, 033026 (2008).

[36] C. Gahl, A. Azima, M. Beye, M. Deppe, K. Döbrich, U. Hasslinger, F. Hennies, A. Melnikov, M. Nagasono, A. Pietzsch, M. Wolf, W. Wurth, and A. Föhlisch, A femtosecond $\mathrm{x}$ ray/optical cross-correlator, Nat. Photon. 2, 165 (2008).

[37] B. Ziaja, N. Medvedev, V. Tkachenko, T. Maltezopoulos, and W. Wurth, Time-resolved observation of band-gap shrinking and electron-lattice thermalization within x-ray excited gallium arsenide, Sci. Rep. 5, 18068 (2015).

[38] M. Harmand, R. Coffee, M. Bionta, M. Chollet, D. French, D. M. Zhu, D. T. Fritz, H. Lemke, N. Medvedev, B. Ziaja, S. Toleikis, and M. Cammarata, Achieving few-femtosecond time-sorting at hard x-ray free-electron lasers, Nat. Photon. 7, 215 (2013).

[39] R. Riedel, A. Al-Shemmary, M. Gensch, T. Golz, M. Harmand, N. Medvedev, M. Prandolini, K. Sokolowski-Tinten, S. Toleikis, U. Wegner, B. Ziaja, N. Stojanovic, and F. Tavella, Single-shot pulse duration monitor for extreme ultraviolet and X-ray freeelectron lasers, Nat. Commun. 4, 1731 (2013).

[40] V. Tkachenko, N. Medvedev, Z. Li, P. Piekarz, and B. Ziaja, Transient optical properties of semiconductors under femtosecond x-ray irradiation, Phys. Rev. B 93, 144101 (2016). 
[41] F. Trani, G. Cantele, D. Ninno, and G. Iadonisi, Tight-binding calculation of the optical absorption cross section of spherical and ellipsoidal silicon nanocrystals, Phys. Rev. B 72, 075423 (2005).

[42] N. D. Mermin, Lindhard dielectric function in the relaxationtime approximation, Phys. Rev. B 1, 2362 (1970).

[43] G. Röpke, A. Selchow, A. Wierling, and H. Reinholz, Lindhard dielectric function in the relaxation-time approximation and generalized linear response theory, Phys. Lett. A 260, 365 (1999).

[44] Y. V. Arkhipov, A. B. Ashikbayeva, A. Askaruly, A. E. Davletov, and I. M. Tkachenko, Dielectric function of dense plasmas, their stopping power, and sum rules, Phys. Rev. E 90, 053102 (2014).

[45] N. Ashcroft and N. Mermin, Solid States Physics (Rinehart and Winston Holt, New York, 1976).

[46] L. Van Hove, Correlations in space and time and Born approximation scattering in systems of interacting particles, Phys. Rev. 95, 249 (1954).

[47] N. H. March and M. P. Tosi, Atomic Dynamics in Liquids (Courier, North Chelmsford, MA, 1991).

[48] A. Volkov and V. Borodin, Heating of metals in swift heavy ion tracks by electron ion energy exchange, Nucl. Instrum. Methods Phys. Res. Sect. B 146, 137 (1998).

[49] C. Ringhofer, M. Nedjalkov, H. Kosina, and S. Selberherr, Semiclassical approximation of electron-phonon scattering beyond Fermi's golden rule, SIAM J. Appl. Math. 64, 1933 (2004).

[50] J. C. Tully, Molecular dynamics with electronic transitions, J. Chem. Phys. 93, 1061 (1990).

[51] J. C. Tully, Perspective: Nonadiabatic dynamics theory, J. Chem. Phys. 137, 22A301 (2012).

[52] C. Zhu, S. Nangia, A. W. Jasper, and D. G. Truhlar, Coherent switching with decay of mixing: An improved treatment of electronic coherence for non-Born-Oppenheimer trajectories, J. Chem. Phys. 121, 7658 (2004).

[53] S. C. Cheng, C. Zhu, K. K. Liang, S. H. Lin, and D. G. Truhlar, Algorithmic decoherence time for decay-of-mixing non-BornOppenheimer dynamics, J. Chem. Phys. 129, 024112 (2008).
[54] N. Medvedev, Femtosecond x-ray induced electron kinetics in dielectrics: Application for FEL-pulse-duration monitor, Appl. Phys. B 118, 417 (2015).

[55] T. G. White, N. J. Hartley, B. Borm, B. J. B. Crowley, J. W. O. Harris, D. C. Hochhaus, T. Kaempfer, K. Li, P. Neumayer, L. K. Pattison, F. Pfeifer, S. Richardson, A. P. L. Robinson, I. Uschmann, and G. Gregori, Electron-Ion Equilibration in Ultrafast Heated Graphite, Phys. Rev. Lett. 112, 145005 (2014).

[56] J. J. Sakurai and J. Napolitano, Modern Quantum Mechanics, 2nd ed. (Pearson New International, Essex, 2014).

[57] N. A. Medvedev, H. O. Jeschke, and B. Ziaja, Non-thermal phase transitions in semiconductors under femtosecond XUV irradiation, SPIE Proc. 8777, 877709 (2013).

[58] J. Gaudin, N. Medvedev, J. Chalupský, T. Burian, S. DastjaniFarahani, V. Hájková, M. Harmand, H. O. Jeschke, L. Juha, M. Jurek, D. Klinger, J. Krzywinski, R. A. Loch, S. Moeller, M. Nagasono, C. Ozkan, K. Saksl, H. Sinn, R. Sobierajski, P. Sovák, S. Toleikis, K. Tiedtke, M. Toufarová, T. Tschentscher, V. Vorlíček, L. Vyšín, H. Wabnitz, and B. Ziaja, Photon energy dependence of graphitization threshold for diamond irradiated with an intense XUV FEL pulse, Phys. Rev. B 88, 060101(R) (2013).

[59] M. Beye, F. Sorgenfrei, W. F. Schlotter, W. Wurth, and A. Föhlisch, The liquid-liquid phase transition in silicon revealed by snapshots of valence electrons, Proc. Natl. Acad. Sci. USA 107, 16772 (2010).

[60] M. Harb, R. Ernstorfer, T. Dartigalongue, C. T. Hebeisen, R. E. Jordan, and R. J. D. Miller, Carrier relaxation and lattice heating dynamics in silicon revealed by femtosecond electron diffraction, J. Phys. Chem. B 110, 25308 (2006).

[61] M. Harb, R. Ernstorfer, C. T. Hebeisen, G. Sciaini, W. Peng, T. Dartigalongue, M. A. Eriksson, M. G. Lagally, S. G. Kruglik, and R. J. Miller, Electronically Driven Structure Changes of Si Captured by Femtosecond Electron Diffraction, Phys. Rev. Lett. 100, 155504 (2008).

[62] S. K. Sundaram and E. Mazur, Inducing and probing nonthermal transitions in semiconductors using femtosecond laser pulses, Nat. Mater. 1, 217 (2002). 